\title{
Multilingualization Experiment of Geographical Name Information Using Vector Title Format
}

\author{
Masaki SUGA $^{\text {a, }}{ }^{\text {, Koji OTSUKA }}{ }^{\text {a, }}$ Yoshikazu HONDA ${ }^{\text {a }}$ \\ ${ }^{a}$ Geospatial Information Authority of Japan, National Mapping Department, Geographical Name Information Division, \\ suga-m96rd@mlit.go.jp \\ * Corresponding author
}

\begin{abstract}
Multilingualization of geographical name information has been required to support foreign visitors to the Olympic Games and the Paralympic Games to be held in Tokyo in 2020 and to correspond to the rapid increase of foreign visitors in recent years. GSI has prepared geographical name information in English and it has been already released. To expand this effort, we have conducted the experiment to translate geographical name information in Japanese and English into French, Korean, Simplified Chinese, and Traditional Chinese automatically and to prepare a multilingual map using vector tile format. To be specific, we prepared transformation rules for translation of geographical name information, and implemented the Javascript codes of transformation rules to deploy in combination with the existing Javascript library group which controls display on the web map. Thus, we developed a web map system which automatically generates geographical name information in these targeted languages on web browsers.
\end{abstract}

Keywords: multilingualization, geographical name information, vector tile data, web map system

\section{Introduction}

Geospatial Information Authority of Japan (GSI) has been developing various data related with national land of Japan, and providing it in an easy-to-use manner, as the national surveying and mapping organization of Japan. As for geographical name information, GSI has developed the geographical names database that is based on the feature names listed in the topographic maps of the scale of $1: 25,000$. GSI develops various geoinformation based on this database and provides it through the Web Map of GSI (GSI Maps), and the database enables GSI Maps to search for location information by geographical names.

Recently, multilingualization of geographical name information has been required to support foreign visitors to the Olympic and Paralympic Games to be held in Tokyo in 2020 and to corresponding to the rapid increase of foreign visitors (Fig.1). GSI has prepared geographical name information in English and it has been already released. Therefore, to expand this effort, we have conducted the experiment to translate geographical name information in Japanese and English into French, Korean, Simplified Chinese, and Traditional Chinese automatically and to prepare a multilingual map using vector tile format. English and these targeted languages covers about $85 \%$ of foreign visitors in the point of their understandable languages as of 2017 in the figure of Japan National Tourism Organization (JNTO).

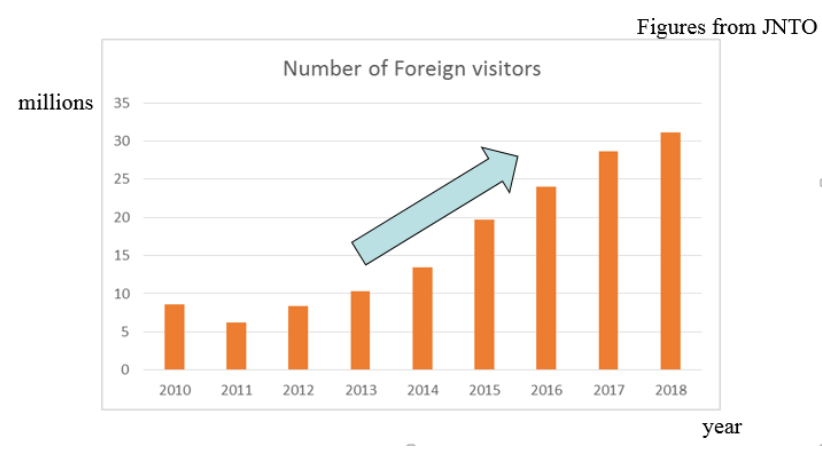

Figure 1. Number of foreign visitors to Japan: Figures from Japan National Tourism Organization (JNTO).

\section{Preparation of transformation rules}

Firstly, we divided the targeted languages into two groups whose structures are similar, which are 1) English and French, and 2) Japanese, Korean, Simplified Chinese, and Traditional Chinese. In addition to it, we arranged the simple transformation rules for translation, which transform 1) English into French, and 2) Japanese into Korean, Simplified Chinese, and Traditional Chinese. To be specific, we divided each geographical name information into proper noun part (original name of its own: e.g. "Fuji" in Mt. Fuji) and generic term part (common name shared by the same entity of geographical features: e.g. "Mt.” in Mt. Fuji) in the original language, and transform the each part into targeted language. Specific methods are as follows.

\subsection{French}

We prepared transformation rules for generic terms by ourselves referencing from "Notation Rules of 
Geographical Names etc. in English” by GSI by translating the generic English terms into the corresponding French names. In addition to it, famous ocean and sea names are prepared referencing from the web map from "IGN France”.

\subsection{Korean}

We prepared the transformation rules for generic terms using "Toponymic Guidelines for Map and Other Editors For International Use” by National Geographic Information Institute. Conversion rules of Japanese Hiragana characters into Korean characters are referenced from “Foreign Language Notation” by National Institute of Korean Language.

\subsection{Simplified Chinese}

We prepared transformation rules for generic terms using "Transformation guidelines of geographical names from foreign language into Chinese - Part 10: Japanese” by China Toponymy Research Institute. Conversion rules of Japanese Kanji characters into Simplified Chinese characters are referenced from "Kanconvit" by Tatsuo YAMASHITA.

\subsection{Traditional Chinese}

We prepared transformation rules for generic terms using "Transformation guidelines of geographical names from foreign language into Chinese - Part 10: Japanese” by China Toponymy Research Institute as well. Conversion rules of Japanese Kanji characters / Simplifed Chinese chracters into Traditional Chinese characters are referenced from "Comparison Table: Traditional Chinese, Simplified Chinese, New Character forms of Kanji” by Masahiko SATO.

\section{Experiment of the translation on a web map}

We also developed Javascript codes (Fig. 2) to implement the transformation rules and deployed them in combination with the existing Javascript library group which controls display on the web map. Thus, we developed a web map system which automatically generates geographical name information in other languages on web browsers. The web map is experimentally published and can be viewed as a map displaying geographical names in multiple languages (Fig.3). The web map system has voice reading function in Japanese for foreigners unfamiliar with Japanese language and pop-up explanation by clicking the annotation or the symbol on the web map.
GSI.GLOBALS.toKr31X = function(word, sound) \{

var es = sound.substr(sound.length - 2, 2);

var esk = "やまさんざんたけだけ";

if (esk.indexOf(es) >=0)\{

esk = "산";

\}

else \{

$$
\begin{aligned}
& \text { es = "'; } \\
& \text { esk = ""; }
\end{aligned}
$$

\}

Figure 2. Part of the Javascript code for transformation (e.g. Korean).

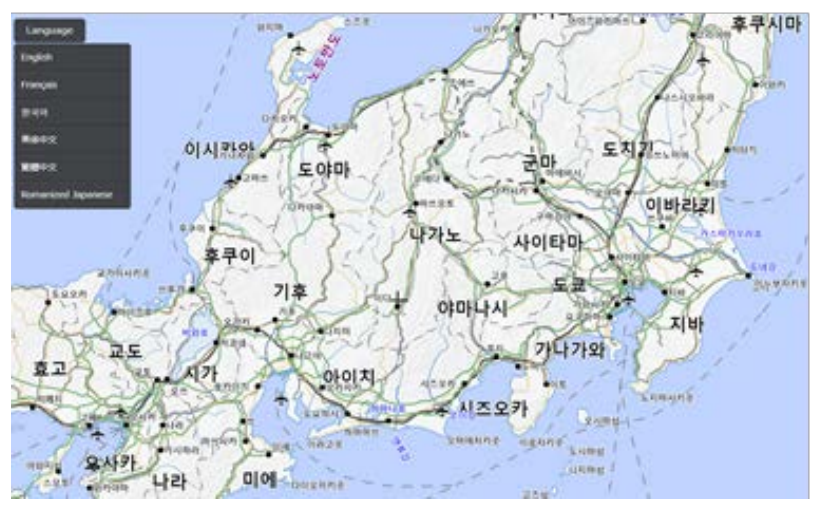

Figure 3. Experiment in the multilingualization on a web map (e.g. Korean).

\section{Conclusion}

We developed a multilingual web map by adopting the automatic translation which consists of simple transformation rules. It should be pointed out that the generated geographical name information in each language is not necessarily accurate especially because of the incompleteness of transformation rules of generic terms. In some Japanese geographical name information, there are no parts corresponding to the generic terms explicitly, and we transliterate them in other languages. However, we expect to contribute smoother communication for foreign visitors who are unfamiliar with the geographical name information written in Japanese, when they can grasp their current and destination place names in their understandable languages. GSI will continue to make efforts to provide geographical name information in an easy-to-use manner.

\section{References}

China Toponymy Research Institute: Transformation guidelines of geographical names from foreign language into Chinese - Part 10: Japanese (Draft)

Geospatial Information Authority of Japan: Notation Rules of Geographical Names etc. in English (2016) http://www.gsi.go.jp/common/000138865.pdf (accessed on March 27th)

IGN France https://www.geoportail.gouv.fr/ (accessed on March 27th) 
JNTO https://www.jnto.go.jp/jpn/statistics/visitor_trends/ (accessed on March 27th)

National Geographic Information Institute: Toponymic Guidelines for Map and Other Editors For International Use

http://www.ngii.go.kr/en/contents/contentsView.do?rbsI $\mathrm{dx}=81$ (accessed on March 27th)

National Institute of Korean Language: Foreign Language Notation

https://www.korean.go.kr/front/page/pageView.do?page _id=P000108\&mn_id=97 (accessed on March 27th)

https://www.korean.go.kr/front/page/pageView.do?page _id=P000129\&mn_id=97 (accessed on March 27th)

Masahiko SATO: Comparison Table: Traditional Chinese, Simplified Chinese, New Character forms of Kanji

http://jgrammar.life.coocan.jp/ja/tools/ksimple.htm (accessed on March 27th)

Tatsuo YAMASHITA: Kanconvit

https://chalow.net/2009-06-14-1.html (accessed on March 27th) 